\title{
CARTILHA DE PASSATEMPOS COMO VEÍCULO DE EDUCAÇÃO EM SAÚDE BUCAL
}

\section{PUZZLE BOOK AS AN EDUCATION TOOL FOR ORAL HEALTH}

\section{Andressa Secco* Marceli Dias Ferreira ${ }^{* *}$ Alessandra de Souza Martins ${ }^{* * *}$ Cristina Berger Fadel ${ }^{* * * *}$}

\section{RESUMO}

O lúdico é uma estratégia cada vez mais utilizada para transmitir valores e impulsionar mudanças de comportamento na área da saúde. Este trabalho, vinculado ao projeto de extensão Nós na Rede, objetiva apresentar a utilização de uma cartilha de passatempos como meio de sensibilização e motivação infantil no campo da saúde bucal. A cartilha intitulada 'Vamos colorir?' vem sendo empregada como meio auxiliar do acesso ao conhecimento infantil, abordando os temas etiologia e prevenção da cárie dentária, com ênfase no papel da dieta, na susceptibilidade à doença e na orientação em higiene bucal. Direcionada para crianças em fase de alfabetização, apresenta 14 passatempos inéditos com atividades para colorir, jogo dos sete erros, liga pontos, caça ao tesouro, labirinto e palavras cruzadas. Esse instrumento é utilizado em eventos de saúde pontuais e de livre demanda e vem sugerindo o pronto efetivar da sensibilização e da facilitação do acesso infantil a informações de saúde bucal.

Palavras-chave- criança; saúde bucal; educação em saúde; materiais educativos e de divulgação; odontologia.

\section{ABSTRACT}

The ludic strategy is used by health professionals as a tool capable of transmitting values and promoting changes in behavior. The aim of this study, which is linked to the extension project 'Nós na Rede,' is to

\footnotetext{
* Aluna de graduação da Universidade Estadual de Ponta Grossa (UEPG), Paraná - Brasil. andressa_3556@hotmail.com

** Aluna de graduação da Universidade Estadual de Ponta Grossa (UEPG), Paraná - Brasil. marcelif23@gmail.com

*** Professora da Universidade Estadual de Ponta Grossa (UEPG), Paraná - Brasil. alessandraphn@hotmail.com 
present the use of a puzzle book as a means of raising awareness of and motivating oral health. The booklet entitled 'Vamos colorir?' has been used as support material to access children's knowledge about the etiology and prevention of tooth decay, emphasizing the role of diet, susceptibility to the disease, and guidance on oral hygiene. This book, which is directed to children in the literacy phase, presents 14 entertaining games, including drawings to be colored, spot the seven errors, connect the dots, treasure search, labyrinth, and crosswords. This instrument is utilized in health events at free demand, and it has been shown to be an effective tool to raise awareness and to facilitate the access to oral health information by children.

Keywords: child; oral health; education in health, educational and dissemination materials; dentistry.

\section{Introduçáo}

Atualmente, a interação entre ludicidade e saúde vem ocupando importante espaço de discussão na área educacional, rompendo o paradigma de que brincadeira seria somente um objeto isolado de reflexão infantil. Autores afirmam que o brincar constitui-se atividade indispensável para o desenvolvimento humano, visando a socialização, a interação social e a aprendizagem.(OLIVEIRA, 2014; GINSBURG et al., 2007; GOLDSTEIN, 2012; DA SILVA et al.,2016).

Em especial, no contexto infantil, o desenvolvimento de formas motivacionais mais sensíveis representa elemento central no estabelecimento da comunicação e, consequentemente, no processo de ensino e aprendizagem. A criança é um sujeito social e histórico, inserida em uma sociedade, com uma determinada cultura, e o lúdico representa uma ponte para a aquisição de novos valores e conhecimentos.Portanto, jogos e brincadeiras, carregados de simbologias,são como exercícios de (re) elaboração e construção de comportamentos e de descobertas de novos caminhos para a aprendizagem. (DALLABONA; MENDES,2004; MOYLES, 2002).

Ojeito de fazer saúde acumulado tradicionalmente nas formas populares de cuidar, denominadas práticas populares de cuidado, tem desvelado possibilidades de construção de processos de cuidado dialogados, participativos e humanizados, acolhedores da cultura e do saber popular. (BRASIL, 2012). No universo da educação e saúde bucal, a vivência de experiênciassignificativas pode favorecer o envolvimento da criança na construção de novos conhecimentos, facilitando a mudança de atitudes, hábitos e cuidados .

Cientes da relevância da efetivação de práticas educativas em saúde por meio da ludicidade, este trabalho se propóe a apresentar a utilização de uma cartilha de passatempos como meio de sensibilização e motivação infantil no campo da saúde bucal.

\section{Referencial teórico-metodológico}

A presente iniciativa está vinculada ao projeto de extensão 'Nós na Rede: Contribuiçóes da Odontologia para Educação, Prevenção e Manutenção da Saúde’, do Departamento de Odontologia da Universidade Estadual de Ponta Grossa (UEPG), e conta com a participação de acadêmicos graduandos e pós-graduandos, agentes universitários e professores.

O referido projeto atua como viabilizador da inserção social em busca da quebra do paradigma do ensino reprodutivo e descontextualizado, envolvendo-se em novas formas de produçáo do conhecimento e aplicação social, com ênfase na estratégia política e metodológica nacional denominada Promoção da Saúde. (BRASIL, 2006; DE ALMEIDA et al., 2012; GUIMARÃES, 2003). Essa é uma das estratégias 
do setor de saúde que trabalha na perspectiva da integralidade de saberes e práticas, pois proporciona o encontro com outros espaços, com outros agentes e com tecnologias que qualificam a relação entre os cidadãos, uma vez que se pauta na subjetividade inerente aos seres humanos. Suas açôes são consolidadas em diversos espaços sociais, em órgãos definidores de políticas e, também, nas universidades, buscando a formação de redes de apoio ao desenvolvimento comunitário. (NARVAI, 2003).

O projeto 'Nós na Rede' desenvolve práticas voltadas à comunidade do município de Ponta Grossa/PR e regiáo adscrita, por meio de parcerias com equipamentos públicos, privados e instituiçóes sem fins lucrativos. Suas açôes são desenvolvidas em diversos espaços sociais, como áreas de lazer, praças, centros esportivos, feiras populares ou qualquer outro ambiente com potencialidade para a promoção da saúde (bucal).(BORDIN et al., 2012).

A cartilha de passatempos intitulada 'Vamos colorir?' é direcionada para crianças em fase de alfabetizaçáo, em que a construçáo da curiosidade é mais explorada na tentativa natural de conhecer o mundo. Sua finalidade éincitar a descoberta de informaçóes relacionadas à saúde bucal, visando favorecer o envolvimento infantil na construção de práticas e comportamentos saudáveis. Além disso, esse instrumento busca suprir a carência de políticas de apoio voltadas à comunidade local, no âmbito da saúde bucal infantil, e propiciar ao acadêmico extensionista de Odontologia uma nova vivência prática da atenção coletiva em saúde.

Essa cartilha apresenta 14 passatempos inéditos, abordando os temas etiologia e prevenção da cárie dentária, com ênfase no papel da dieta e a susceptibilidade à doença e na orientaçáo sobre higiene bucal. As atividades que compóem esse instrumento foram confeccionadas com figuras disponibilizadas pela página virtual de acesso gratuito Freeimages (http://pt.freeimages.com/) e adaptadas à lógica da educação em saúde bucal.

Para mobilizar os processos de aprendizagem infantil de modo a corroborar no desenvolvimento de suas capacidades relacionadas à leitura e à escrita e na construçáo de representaçóes sobre o objeto de estudo, as situaçóes de aprendizagem criadas foram desenvolvidas de forma sequenciada, articulada e contextualizada. (ALEXANDER, 2000; GRIFFIN et al., 2003). Os passatempos foram também pensados sob um enfoque de clareza e atratividade, com figurasimpressas em preto e branco e de tamanho que possibilite pinturapara que, com a resolução das atividades, a criança possa se sentir motivada a conquistar e manter um sorriso saudável. Além da atividade de colorir, há também o jogo dos sete erros, liga pontos, caça ao tesouro, labirinto e palavras cruzadas (Figura 1).

A atividade lúdica vem sendo cada vez mais utilizada por profissionais dediferentes áreas da saúde, uma vez que serve de estímulo para a construçáo do conhecimento humano, constituindo-se importante ferramenta de desenvolvimento pessoal, capaz de transmitir valores e, até mesmo, impulsionar mudanças no comportamento, principalmente de crianças. (DA SILVA et al., 2016; HAYES, 2003; HOWARD, 2010; MCINNES et al., 2011). As crianças, quando brincam ou realizam atividades prazerosas, buscam atribuir significados e estabelecer vínculos, tornando-se assim capazes de construir aprendizados. (VYGOTSKY, 1998; MCARDLE, 2001; LINSEY, 2003). Dessa forma, é na interaçáo com as atividades que envolvem simbologias que a criança aprende a agir numa esfera cognitiva, possibilitando a ampliaçáo de sua consciência e aconstrução de sua autonomia. 


\section{Figura 1- Representaçáo gráfica da cartilha 'Vamos Colorir?’. UEPG. Ponta Grossa/PR, 2016}
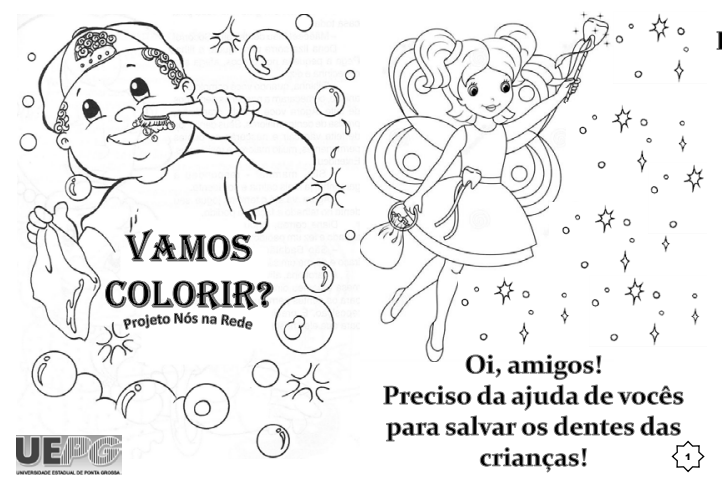

Preciso da ajuda de vocês para salvar os dentes das crianças! 访

Como é bom ir ao dentista!

\section{Quando comemos e não escovamos os dentes, a} cárie aparece!
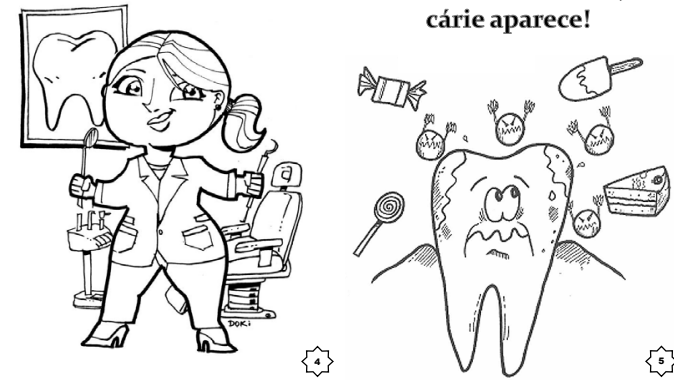

Ligue os pontos e descubra Vamos ver se você é bom em nosso melhor aliado! achar os 7 erros?

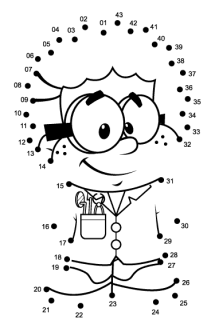

Dentista A) para o combate aos monstros da cárie!

mara queos monstros da cárie não machuquem os dentes, estes três
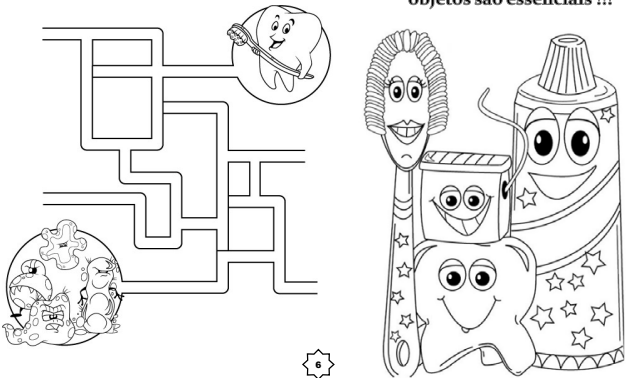
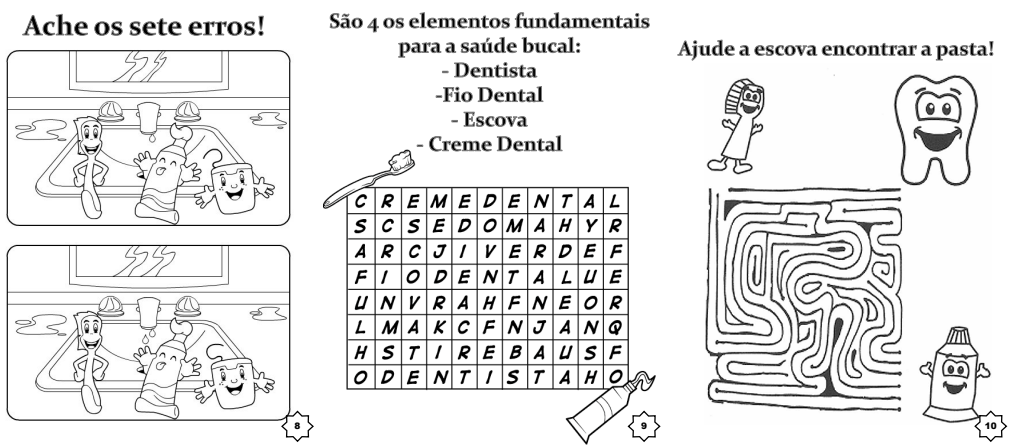

Existem 7 erros nestas imagens.

Você sabe quais são?

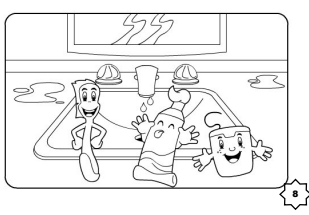

Ligue os pontos e
descubra o desenho!

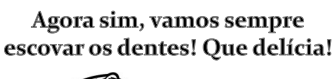

\section{(3)}
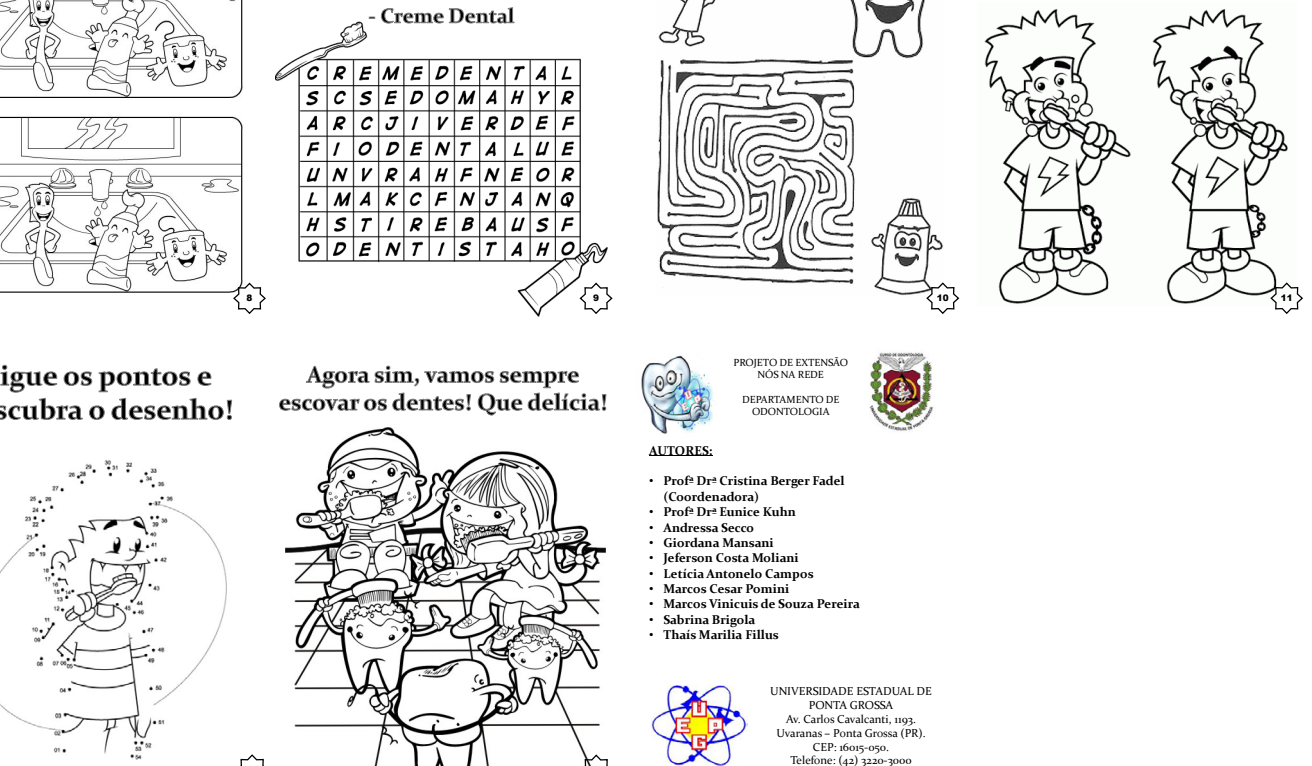

$\widehat{\{12}\}$

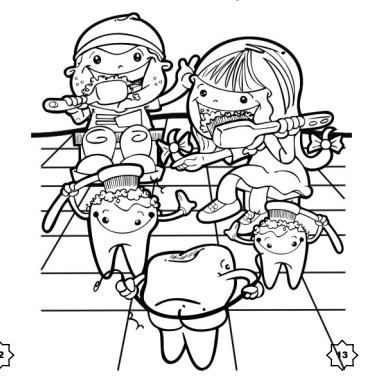

AUTORES:

Prof Dre Cristina Berger Fadel
(Coordenadora)

. (Coordenadora)

: Andressa Secco
Giordana Mansani

Jeferson Costa Moliani

Marcos Cesar Pomini

Sabrina Brigola

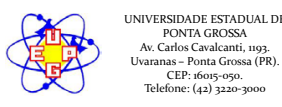

Quanto à forma de utilização da cartilha, esse instrumento vem sendo empregado como meio auxiliar do acesso ao conhecimento, frequentemente sucedendo um diálogo individual ou coletivo sobre o tema saúde bucal (Figuras 2 e 3). Com o emprego dessa cartilha, verificou-se a importância de propostas prévias de contato dos sujeitos com o mote a ser explorado, ainda que de baixa complexidade, para que exista realmente um valor significativo na ação, enquanto prática educacional e pedagógica. Essa condição de 
métodos conjuntos torna-se importante fator motivacional para crianças, uma vez que propicia reforço a conhecimentos prévios ou já existentes e estímulo à exploração de sua criatividade.

Figura 2- Utilizaçáo da cartilha 'Vamos Colorir?'em instituiçáo educacional pública, como meio motivacional após orientação sobre higiene bucal. Ponta Grossa/PR, 2016

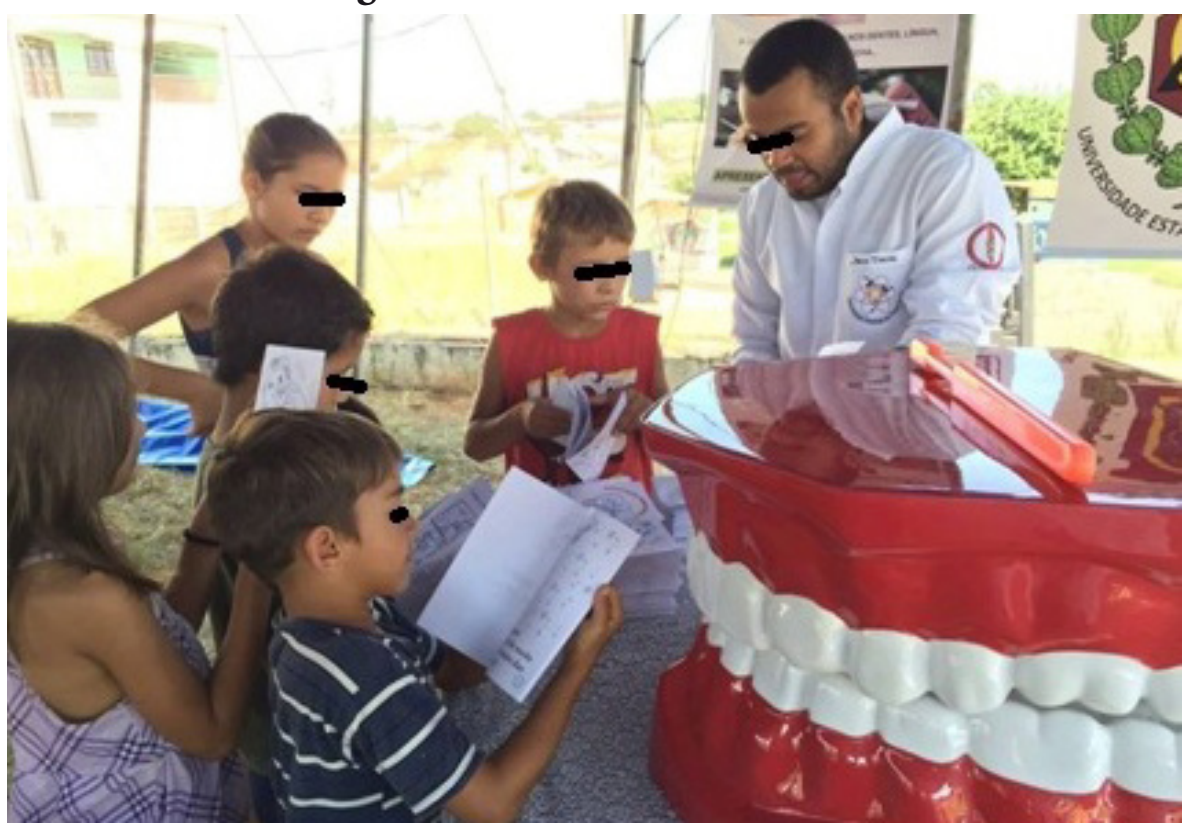

Figura 3- Utilizaçáo da cartilha 'Vamos Colorir?'.em praça pública, como meio motivacional após roda de conversa sobre saúde bucal. Ponta Grossa/PR, 2016

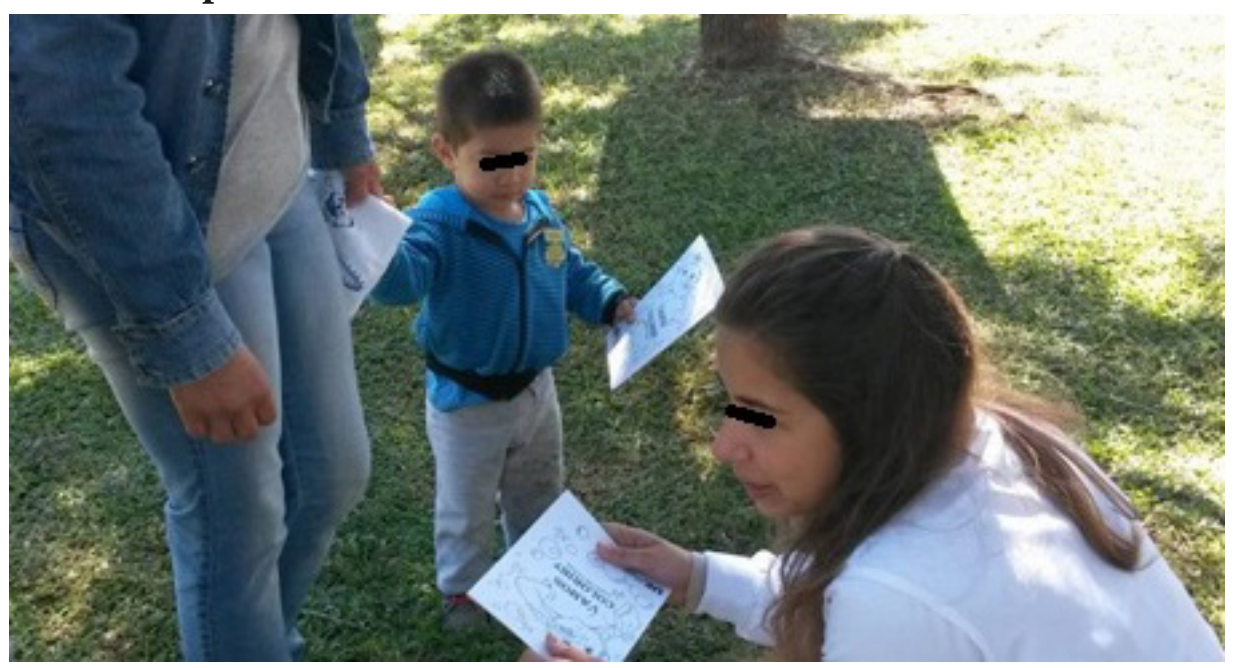

\section{Resultados e Discussão}

A educaçáo em saúde tem sido extremamente bem sucedida na motivaçáo e modificação de comportamentos de saúde, como demonstrado por KAY e LOCKER (1998) e WATT (2005). Tradicionalmente, sabe-se que a base para intervençôes mais profícuas em saúde deve estar ancorada no reconhecimento biológico dos sujeitos e, modernamente, também em seu contexto social. (EMMONS, 2000). 
No entanto, a prática aqui proposta, de utilizaçáo de uma cartilha de passatempos em eventos de saúde pontuais e de livre demanda, vem sugerindo o pronto efetivar da sensibilização e da facilitação do acesso infantil a informaçōes de saúde bucal. Crianças de pequena idade tendem a reagir de forma rápida e positiva à exposição de novas informaçôes, sem a necessidade de profunda contextualização de saberes (BROTHERSON, 2005), e essa afirmação pode justificar os resultados satisfatórios alcançados com a cartilha.

Até o momento, o referido instrumento foi utilizado com outras medidas educativas, atingindo aproximadamente 750 crianças, com idade de 5 a 10 anos, que expuseram alteraçáo quase imediata do interesse em relaçáo à saúde bucal. Dentre elas, as situaçóes mais observadas foram: curiosidade sobre a etiologia da doença cárie e perda dos dentes decíduos, vontade de praticar repetidamente escovação dentária e lingual, desejo em demonstrar aos extensionistas os novos conhecimentos adquiridos e comprometimento em utilizar a cartilha como reforço de aprendizagem e motivaçáo em seu núcleo familiar.

$\mathrm{Na}$ condição de sujeitos sociais, inseridos em manifestaçôes históricas e hábitos culturais, as crianças em tela, frequentemente, apresentam-se acompanhadas de pais, responsáveis ou cuidadores. Esse aspecto é levado em consideração quando da utilização da cartilha 'Vamos Colorir?', frente à ciência de que a participaçáo efetiva de pais ou responsáveis nos processos lúdicos educativos tende a transformar a aprendizagem num processo ativo, em que a informação transmitida é colocada em prática e o fator divertimento traz novas sensaçóes e aproximaçóes sociais.

Outro facilitador do desencadeamento de forças motivadoras no instrumento proposto se refere ao fator idade. Estudos mostram que as práticas de educaçáo em saúde devem ser expostas à população o mais precocemente possível (PELOSI, 2000; WATSON et al., 2001; SALIBA et al., 2003), sendo a faixa etária ideal para se fomentar hábitos saudáveis e para a participaçáo em programas educativo-preventivos de saúde bucal, de dois a nove anos. (BRASIL, 2006; VARGAS; LOBATO, 2007). Os métodos educativos devem ser utilizados com o intuito de tornar a aprendizagem mais agradável, atraente, significativa e estimulante, principalmente quando se trabalha com a população infantil. (ANTUNES et al., 2006; MASTRANTONIO; GARCIA, 2002; SANTOS,2009; RODRIGUES, 2003; LINSEY). Nesse sentido, a cartilha 'Vamos Colorir?'vem cumprindo importante papel de aproximaçáo afetiva com as crianças, condiçáo fundamental para o alcance dos objetivos desejados. Ao despertar a curiosidade infantil, seu significado pode ser discutido de forma descontraída e prazerosa e o conhecimento provocado, a partir da atividade lúdica, pode ser transportado para o campo da realidade, caracterizando a transformaçáo social.

Desse modo, a utilizaçáo do recurso pedagógico exposto vem apresentando resultados bastante expressivos em todas as comunidades, uma vez que consegue atrair a atenção individual e coletiva para os temas de interesse, despertando a curiosidade, auxiliando na (re)definição de valores, no processo de autonomia em saúde e na motivação para a aquisiçẫo e a manutenção da saúde bucal.

\section{Conclusão}

Diante do exposto, conclui-se que a estratégia de educação em saúde proposta pela cartilha 'Vamos Colorir?' é de extrema relevância social, uma vez que impulsiona a atividade lúdica como meio facilitador do compartilhamento de informaçôes e da motivação de crianças em busca de condutas mais saudáveis no campo da saúde bucal. 


\section{Referências}

ALEXANDER, R. E. Readability of published dental educational materials. The Journal of the American Dental Association, v. 131, n. 7, p. 937-942, 2000.

ANTUNES,L. D. S.;et al. Avaliação da percepção das crianças e conhecimento dos educadores frente à saúde bucal, dieta e higiene. Pesqui. bras.odontopediatria clín. integr, v.6, n.1, 79-85, 2006.

BORDIN, D.; BORDIN, R.; FADEL.C.B.Projeto de extensão'Nós na Rede': a odontologia à luz da promoção da saúde. Revista Conexão UEPG, v. 8, n. 1, p. 86-93, 2012.

BRASIL. Ministério da Saúde. Cadernos de Atenção Básica. Saúde Bucal. Departamento de Atenção Básica. Brasília, 2006.

BRASIL. Ministério da Saúde. Caderno de Educação Popular e Saúde. Secretaria de Gestão Estratégica e Participativa. Departamento de Apoio à Gestão Participativa. Brasília,2012.

BROTHERSON, S. E. Understandingbraindevelopment in youngchildren.NDSU Extension Service, 2005.

DALLABONA, S. R.; MENDES, S. M. S.; O lúdico na educação infantil: jogar, brincar, uma forma de educar. Revista de divulgação técnico-científica do ICPG, v. 1, n. 4, p. 107-112, 2004.

DA SILVA, J. R.S.;et al. Usingtherapeutictoystofacilitatevenipuncture procedure in preschoolchildren. Pediatricnursing, v. 42, n. 2, p. 61, 2016.

DE ALMEIDA, L. E.; PEREIRA, M. N.; SILVEIRA, W. J. Educação em Saúde: uma experiência, uma comprovação. Interagir: pensandoaextensão, n. 10, 2012.

EMMONS, K. M. Health behaviors in a social context. Social epidemiology, p. 242-266, 2000.

GINSBURG, K.R.;etal.The importance of play in promoting healthy child development and maintaining strong parent-child bonds. Pediatrics, v. 119, n. 1, p. 182-191, 2007.

GOLDSTEIN, J.Play in Children's development, health and weel-being. Europe: Toy Industries ofEurope (TIE), p.41, 2012.

GUIMARÃES, G. R. A. Promoção da saúde na escola: a saúde bucal como objeto de saber [Dissertação]. Rio de Janeiro: Escola Nacional de Saúde Pública da Fundação Oswaldo Cruz, 2003.

GRIFFIN, J.; MCKENNA, K.; TOOTH, L. Written health education materials: Making them more effective. AustralianOccupationalTherapyJournal, v. 50, n. 3, p. 170-177, 2003.

HAYES, N. Play, care and learning: creating an integrated curriculum for early childhood education in Ireland. Researching Early Childhood, v. 5, p. 69-81, 2003. 
HOWARD, J. Early years practitioners' perceptions of play: An exploration of theoretical understanding, planning and involvement, confidence and barriers to practice. Educational and Child Psychology, v. 27, n. 4, p. 91, 2010.

KAY, E.; LOCKER, D.A systematic review of the effectiveness of health promotion aimed at improving oral health. Community dental health, v. 15, n. 3, p. 132-144, 1998.

LINSEY, E. W.; COLWELL, M. J. Preschoolers' emotional competence: Links to pretend and physical play. ChildStudyJournal, v. 33, n. 1, p. 39-53, 2003.

MASTRANTONIO, S. S.; GARCIA, P. P. N. S. Programas educativos em saúde bucal: revisão da literatura. J. Bras.Odontopediatr.Odontol.Bebe, v. 5, n. 25, p. 215-22, 2002.

MCARDLE, P. Children's play. Child: Care, Health and Development, v. 27, n. 6, p. 509-514, 2001.

MCINNES, K.; et al. Differences in practitioners' understanding of play and how this influences pedagogy and children's perceptions of play. EarlyYears, v. 31, n. 2, p. 121-133, 2011.

MOYLES, J. R. Só brincar?: o papel do brincar na educação infantil. Artmed, 2002.

NARVAI, P. C. Recursos humanos para promoção da saúde bucal: um olhar no início do século XXI. ABOPREV: promoção de saúde bucal, v. 3, p. 475-94, 2003.

OLIVEIRA, J. C. C. "Atividades lúdicas na Odontopediatria: uma breve revisão da literatura." Revista Brasileira de Odontologia 71.1: 103-107. 2014

PELOSI, M. Psicopedagogia: didática aplicada à Psicopedagogia. UFRJ/CFCH, 2000.

RODRIGUES, D. Educação inclusiva: as boas e as más notícias. Perspectivas sobre a inclusão: da educação à sociedade. Porto: Editora Porto, 2003.

SALIBA, N,A.;et al. Programa de educação em saúde bucal: A experiência da Faculdade de Odontologia de Araçatuba-UNESP. Odontol. clín.-cient, v. 2, n. 3, p. 197-200, 2003.

SANTOS, K. T.Educação em saúde bucal na escola: uma análise dos sujeitos envolvidos no processo. Tese (Doutorado) - Faculdade de Odontologia de Araçatuba, Universidade Estadual Paulista, Araçatuba, 2009.

VARGAS, V. S.; LOBATO, R.C. O desenvolvimento de práticas alimentares saudáveis uma estratégia de educação nutricional no ensino fundamental. 2007.

VYGOTSKY, L.S. A Formação Social da Mente. $6^{\text {a }}$ ed. São Paulo, SP. Martins FontesEditora LTDA, 1998.

WATSON, M. R.;etal.A Community Participatory Oral Health Promotion Program in an Inner-city Latino Community. Journalofpublichealthdentistry, v. 61, n. 1, p. 34-41, 2001.

WATT, R. G. Strategies and approaches in oral disease prevention and health promotion. Bulletinofthe World Health Organization, v. 83, n. 9, p. 711-718, 2005. 